Chirurg 2022 $\cdot 93: 1-2$

https://doi.org/10.1007/s00104-021-01570-y Angenommen: 23. Dezember 2021

(c) The Author(s), under exclusive licence to Springer Medizin Verlag GmbH, ein Teil von Springer Nature 2022

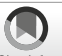

\title{
Herr Professor Büchler als Herausgeber verabschiedet
}

\author{
Er prägte 20 Jahre die Zeitschrift Der Chirurg

\section{C.-T. Germer' · S. Hofmann ${ }^{2}$} \\ 'Zentrum Operative Medizin (ZOM), Klinik für Allgemein-, Viszeral-, Transplantations-, Gefäß- und \\ Kinderchirurgie, Universitätsklinikum Würzburg, Würzburg, Deutschland \\ ${ }^{2}$ Redaktion Der Chirurg, Springer Medizin, Heidelberg, Deutschland
}

Zum Ende des Jahres 2021 hat sich Herr Professor Büchler auf eigenen Wunsch als Herausgeber von Der Chirurg aus dem Board der Zeitschrift zurückgezogen. Er hat die Zeitschrift 20 Jahre lang maßgeblich in unterschiedlichen Funktionen mitgestaltet und geprägt. Bereits im Jahre 2001 wurde Professor Büchler (- Abb. 1) in den Beirat der Zeitschrift berufen. Seit dem Jahr 2007 war Professor Büchler Mitglied im Herausgeber-Board von Der Chirurg.

Herr Professor Büchler gehört zu den renommiertesten Chirurgen weltweit. Er gilt als ausgewiesener Experte in der Allgemein- und Viszeralchirurgie, wobei seine Schwerpunkte die Chirurgie der Bauchspeicheldrüse, die hepatobiliäre Chirurgie und die Transplantationschirurgie darstellen. Er publizierte eine Vielzahl von grundlagenwissenschaftlichen und translationalen chirurgischen Forschungsergebnissen in den renommiertesten internationalen wissenschaftlichen Zeitschriften. Die besondere Bedeutung seiner Forschung wird darin deutlich, dass Herr Professor Büch- ler über viele Jahre Sprecher von DFG-geförderten Transregios, klinischen Forschergruppen und Graduiertenkollegs war. Darüber hinaus entwickelte Professor Büchler neue Operationstechniken, die heute als allgemeingültiger Standard in der Allgemein- und Viszeralchirurgie anerkannt sind.

Herr Professor Büchler hat Medizin in Heidelberg und Berlin studiert und wurde nach seiner chirurgischen Weiterbildung im Jahre 1987 in Ulm habilitiert. Von 1993 bis 2001 war er Professor für Chirurgie und als Klinikdirektor der Abteilung für Viszeral- und Transplantationschirurgie am Universitätsklinikum Bern (Inselspital) in der Schweiz tätig. Nach einer Gastprofessur an der Yale University, School of Medicine, New Haven, USA folgte er im Jahr 2001 dem Ruf an das Universitätsklinikum Heidelberg, um dort die Nachfolge von Herrn Professor Herfarth anzutreten.

Herr Professor Büchler hat eine bedeutende Chirurgenschule etabliert. Seine Schüler bekleiden heute eine Vielzahl

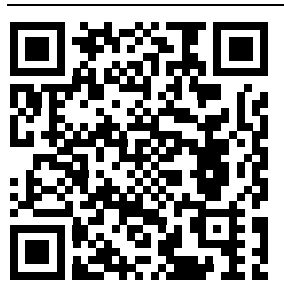

QR-Code scannen \& Beitrag online lesen

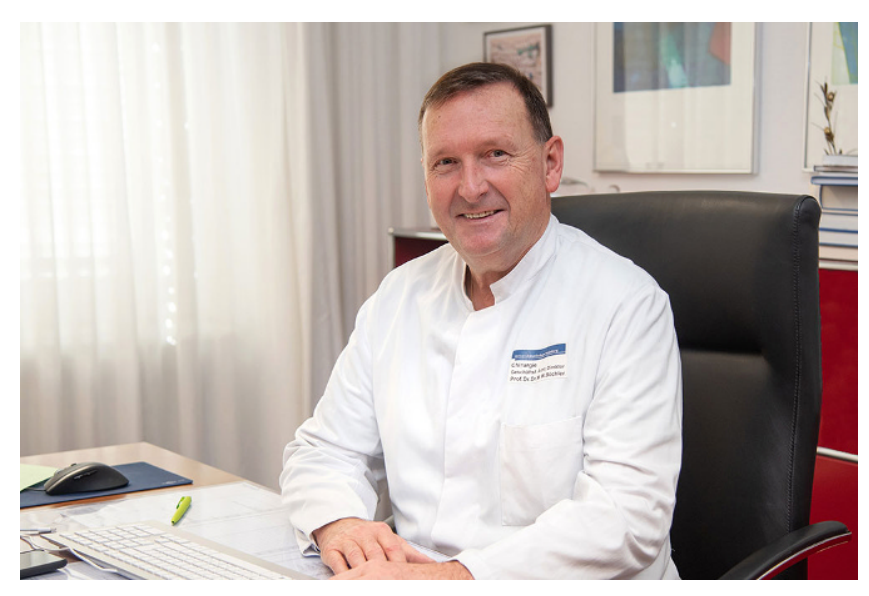

Abb. $1<$ Prof. Dr. med. Dr. h.c. Markus W. Büchler 
von allgemein- und viszeralchirurgischen Lehrstühlen in Deutschland. Die Bedeutung von Professor Büchler für die chirurgische Gemeinschaft wird auch durch die zahlreichen Ehrungen, die ihm im Laufe seiner beruflichen Laufbahn zuteilwurden, verdeutlicht. Alle Ehrungen und Preise an dieser Stelle aufzuzählen würde den Rahmen sprengen. Herr Professor Büchler erhielt viele nationale und internationale Preise, Auszeichnungen, Ehrendoktorwürden und Ehrenprofessuren. Er engagiert sich als Mitglied des Editorial Boards und als Mitherausgeber vieler renommierter Zeitschriften. Seit vielen Jahren ist er auch Editor-in-Chief von Langenbeck's Archives of Surgery. Er wirkte als Vorstandsmitglied und Präsident vieler nationaler und internationaler chirurgischer Fachgesellschaften, so u.a. auch der Deutschen Gesellschaft für Allgemein- und Viszeralchirurgie und der Deutschen Gesellschaft für Chirurgie. Über die Chirurgie hinausweisend ist seine Mitgliedschaft in der renommierten Leopoldina, Nationale Akademie der Wissenschaften.

Herr Professor Büchler hat während seiner Tätigkeit für Der Chirurg eine Vielzahl von Leithemenheften verantwortlich herausgegeben und damit vielfach Standards in der operativen Medizin gesetzt. Die Schriftleitung von DerChirurg sowie die Geschäftsführung und die Redaktion des Springer Medizin Verlags danken Herrn Professor Büchler auf das herzlichste für seinen jahrelangen hohen persönlichen
Einsatz für den Erfolg dieser Zeitschrift. Herr Professor Büchler hat das Gesicht von Der Chirurg nachhaltig geprägt.

Wir wünschen Herrn Professor Büchler für die Zukunft weiterhin Schaffenskraft, Gesundheit und Glück und hoffen auch zukünftig auf seinen Rat und seine Unterstützung bei Der Chirurg bauen zu können.

\section{Prof. Dr. C.-T. Germer}

Schriftleiter Der Chirurg

Sabine Hofmann

Managing Editor Der Chirurg

\section{Korrespondenzadresse}

Univ.-Prof. Dr. med. C.-T. Germer

Zentrum Operative Medizin (ZOM), Klinik für Allgemein-, Viszeral-, Transplantations-, Gefäßund Kinderchirurgie, Universitätsklinikum Würzburg

Oberdürrbacher Str. 6, 97080 Würzburg,

Deutschland

germer_c@ukw.de

Interessenkonflikt. C.-T. Germer und S. Hofmann geben an, dass kein Interessenkonflikt besteht.

\section{DEUTSCHER KREBSKONGRESS}

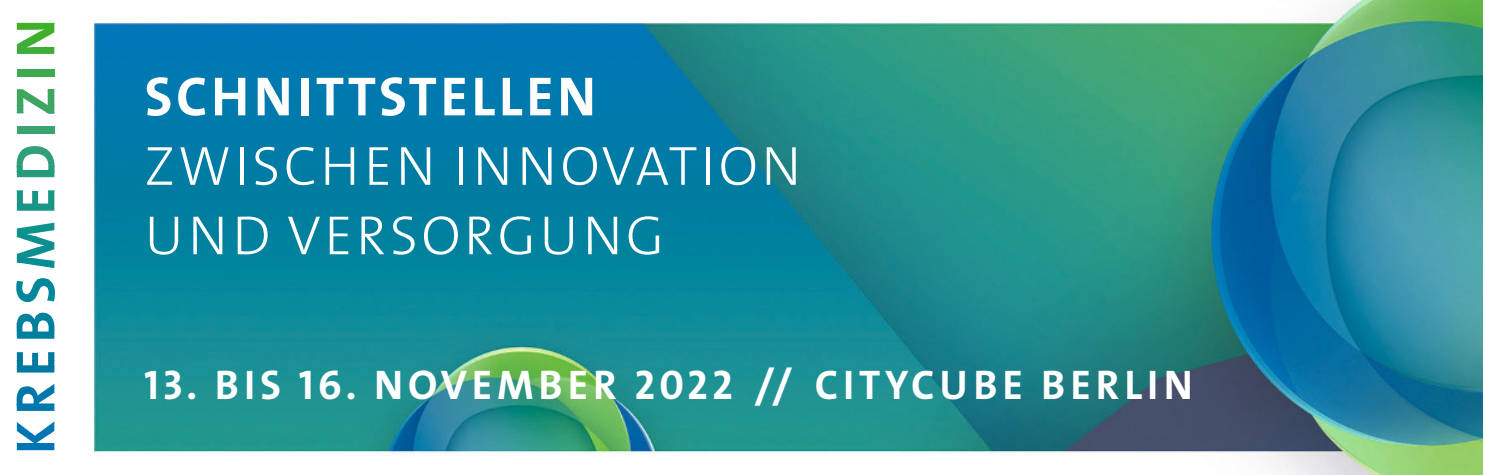

